\title{
Design of a 2D Sparse Array Transducer for Integration into an Ergonomic Transcranial Ultrasound System
}

\author{
Xiaotong Li, Anthony Gachagan, Jerzy Dziewierz, \\ Richard O'Leary \\ Centre for Ultrasonic Engineering \\ University of Strathclyde \\ Glasgow, United Kingdom \\ xiaotong.li@strath.ac.uk
}

\author{
Paul Murray \\ Centre for Signal and Image Processing \\ University of Strathclyde \\ Glasgow, United Kingdom
}

\begin{abstract}
Transcranial Doppler Ultrasound (TCD) is one of the techniques that have been used for stroke diagnosis. This paper compares the potential of three aperiodic sparse array configurations: random array; sunflower spiral array; and log spiral array for application to TCD. To cover the full temporal window, a $30 \mathrm{~mm}$ diameter circular aperture is selected, with a $2 \mathrm{MHz}$ operating frequency to match current TCD instrumentation. A 2D model developed in MATLAB simulates the far field directivity function by applying the 2D FFT on an array's aperture function. Two evaluation criteria, Peak Side-lobe Level (PSL) and Integrated Side-lobe Ratio (ISLR), are used to assess the performance of each array configuration. Simulation results demonstrate that a compromise between PSL and ISLR is required to select a suitable transducer configuration for fabrication and further evaluation. These evaluation results demonstrate that the log spiral array configuration has desirably low PSL relative to the others, while the sunflower spiral array performs better in terms of ISLR. Considering this design evaluation, a prototype array based on a log spiral layout has been manufactured. Characterization of the prototype array show that it performs as predicted.
\end{abstract}

Keywords-2D sparse array; Transcranal Doppler Ultrasoud (TCD); array characterization

\section{INTRODUCTION}

Transcranial Doppler Ultrasound (TCD) is a non-invasive technique that has been widely used in stroke diagnosis and treatment since 1982 [1]. In addition to its non-invasive advantage, it further benefits from portability and low expense of ultrasound devices. However, at present most of the commercial TCD systems use single element transducers or 1D array transducers, which have limited beam steering flexibility and imaging capability [2]. Moreover, these kinds of transducers have to be manually operated by skilled operators during the test. Thus, the results can be highly affected by human factors. Considering these drawbacks, it is anticipated that one way to improve TCD is by using 2D array transducers embedded within an appropriately designed head frame. The 2D array transducers have better imaging capability, while the head frame holds the transducers in position during the TCD test.

Due to the associated half-wavelength limit of element pitch in dense array designs [3], a full matrix 2D array transducer which has comparable resolution to a 1D linear array transducer would require an extremely large number of elements. Unfortunately, this is not practical to manufacture. For the transducer designed in this project, the aperture size is set to 3 $\mathrm{cm}$ in diameter to fit the size of the temporal window [4]. As the speed of sound in the brain is $1550 \mathrm{~m} / \mathrm{s}$ [5], the operating wavelength will be $0.775 \mathrm{~mm}$ if we operate at a $2 \mathrm{MHz}$ center frequency, which is common in such medical applications [2]. If the transducer is designed with element spacing approaching the half-wavelength limit, there would be thousands of elements within the aperture. However, this presents a huge challenge for both manufacturing and controlling the array. One way to reduce the number of elements is by using a 2D sparse array transducer which, if designed correctly, can achieve useful imaging capability while using much fewer elements.

This paper describes the design of a new, bespoke 2D sparse array transducer which could be utilized as part of our proposed ergonomic TCD system. Three sparse array configurations have been studied using a 2D Fast Fourier Transform (FFT) to predict the far-field directivity function of the array configuration. The performance of each proposed array configuration is evaluated using two parameters: Peak Side-Lobe value (PSL) and Integrated Side-Lobe Ratio (ISLR). Based on the simulation results, a log spiral array configuration has been identified as a promising array design and manufactured using a Composite Element Composite Array Transducer (CECAT) fabrication approach [6].

\section{Methodology}

\section{A. Sparse Array Configurations}

Arrays whose element pitch is larger than half the operating wavelength of the device are known as sparse arrays [3]. Compared to dense arrays, sparse arrays are less expensive and easier to fabricate because of the reduced number of elements within the fixed aperture. However, the increased element pitch leads to the introduction of a higher side lobe level which, can result in poorer image contrast. According to the element pattern, sparse arrays can be categorized as, 1) periodic sparse arrays or, 2) aperiodic sparse arrays.

Periodic sparse arrays are easier to design and manufacture, but are more likely to suffer from higher side lobe levels, or form 
grating lobes because of the enhanced interaction between elements [7]. Hence, an aperiodic approach is adopted here.

Three aperiodic sparse array configurations have been considered and simulated: a random array; a sunflower spiral array; and a log spiral array. Figure 1 illustrates examples of these array configurations. The random array configuration is easy to design without forming grating lobes as it breaks the interaction between elements which comes from the periodicity.

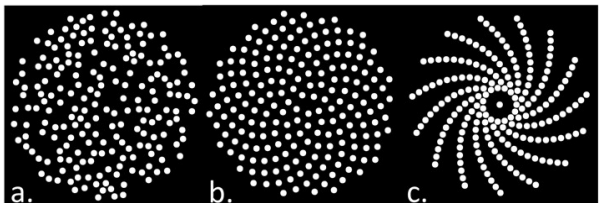

Fig. 1. Three sparse array configurations which have been simulated in this project: (a) random array; (b) sunflower spiral array; and (c) log spiral array.

A random array can be simulated in the following steps:

1) Set the minimum distance (ele_pitch) between array elements to avoid overlapping.

2) Randomly place the desired number of points (N) on a rectangular aperture of $30 \mathrm{~mm}$ dimension.

3) Remove the points which are outside of the $30 \mathrm{~mm}$ diameter circular aperture.

4) Calculate the distance between each pair of points. If the distance is smaller than ele_pitch, remove the point which is generated later.

5) If the number of points remaining in the circular aperture (M) is fewer than $N$, place another $N$ - $M$ points and repeat step 2 to step 4 until $M$ equals to $N$.

The sunflower spiral array is a special Fermat spiral array whose polar angle is equal to integer multiples of the golden angle, $c$ [8]. The mathematical form of the sunflower array is:

$$
r_{n}=a \sqrt{\gamma_{n}}=a \sqrt{n c}, c=2.4 \mathrm{rad}
$$

where $n$ is the $n^{\text {th }}$ point in the sunflower spiral array, $r$ is the radial distance, and $\gamma$ is the polar angle. $a$ is a constant parameter relative to the shape of the sunflower spiral array [8]. The process to generate the sunflower spiral array can be divided into following steps:

1) Set the minimum distance (ele_pitch) between array elements to avoid overlapping.

2) Generate a sunflower array with desired number of elements $(N)$.

3) Find the minimum distance between these elements (ele_pitch $\left.{ }_{\text {real }}\right)$.

4) If ele_pitch real $_{\text {is }}$ not smaller than ele_pitch, the generation process could be stopped. Otherwise, expand the whole array to make them equal.

5) After expansion, remove any points that falls outside of the $30 \mathrm{~mm}$ diameter circular aperture.

A log spiral array which consists of one or several arms can be expressed as:

$$
r=a e^{b(\gamma+\theta)}
$$

Where $r$ and $\gamma$ are the radial distance and the polar angle, respectively. $a$ and $b$ are constant parameters associated with the shape of the array and $\theta$ is the rotational angle between two adjacent arms. As indicated in (2), more parameters are required to generate a log spiral array compared to the other two configurations. Thus, once the parameters are fixed, the layout of log spiral array is fixed. The process can be express as:

1) Set the minimum pitch between array elements along the same spiral arm.

2) Set parameters including: the number of spiral arms within the array; the number of elements within each arm; and the constant paramteter $b$.

3) Generate the log spiral array based on the parameters selected in step 2.

4) Remove the points which fall outside of the $30 \mathrm{~mm}$ circular aperture. Modify $b$ if the elements in the inner circles are overlapped.

5) Place an additional element in the center of the log spiral array to help the orientation of the ultrasound beam while having minimal influence on the PSL and ISLR [7].

The two spiral arrays avoid the periodic pattern of elements while allowing more control over element positioning. The spiral patterns have been studied to form 2D sparse arrays by many projects and achieved positive results [7]-[10].

\section{B. Composite Element Array Composite Transducer (CECAT) Structure}

The Composite Element Composite Array Transducer (CECAT) is a piezocomposite configuration which was designed to improve the flexibility of traditional array transducer technology using conventional 1-3 connectivity composite active layer [6]. Elements in the CECAT configuration are individual piezocomposites, consisting of piezoelectric fiber material embedded in a polymer phase [6]. However, the overall ceramic volume fraction of the array device is lower than a comparable 1-3 piezocomposite array due to the fact that only the array elements contain active piezoceramic material. The piezoelectric fibers are randomly placed within the array element area and the experimental results demonstrate that this random fiber structure can achieve similar performance when compared with the conventional dice-and-fill structure [6]. Furthermore, the random fiber structure can help reduce interpillar modes as it breaks the periodicity of the conventional 1-3 piezocomposite microstructure. The manufacturing process for fiber CECAT is simpler compared to the conventional dice and fill method and more than one active layer can be made from a single manufacturing process [6].

\section{Peak Side-lobe Level (PSL) and Integrated Side-lobe Level (ISLR)}

Two metrics known as, Peak Side-lobe Level (PSL) and Integrated Side-lobe Level (ISLR) are used to estimate the performance of the array configurations in terms of their imaging capability. These two parameters reflect the relative relationship between the main-lobe and the associated sidelobes. PSL is defined as the ratio of the maximum side-lobe value to the maximum main-lobe value. ISLR is defined as the ratio of the total energy contained outside the main-lobe (sidelobes area) to the energy contained inside the main-lobe. These two parameters can be expressed using:

$$
P S L=20 \log _{10}\left(A_{s} / A_{m}\right)
$$




$$
I S L R=10 \log _{10}\left(E_{S} / E_{m}\right)
$$

where $A$ and $E$ stand for the amplitude and the energy in the lobe(s) respectively. The side-lobe is represented by $s$, while the main-lobe is represented by $m$.

Both PSL and ISLR are related to the quality of image which could be achieved by an ultrasonic transducer. They reflect the ability of the imaging system to identify the target in the mainlobe path in the presence of targets or scatters in the side-lobes paths. High PSL or ISLR values could indicate a problem when the target in the main-lobe path is weak [11], or when imaging low contrast materials [7]. Thus, an appropriate transducer should have a low PSL value, as well as low ISLR.

To calculate PSL and ILSR, it is necessary to predict the propagation of ultrasound waves in the imaging material. Method such as the Huygens' theory [12] and the Rayleigh's integral [13] could be used. These methods either assume the piezoelectric element as a point source or an integral of differential planes. Both of them require a large number of operations which could be time consuming. To reduce the operation time, in this work, a 2D Fast Fourier Transform is (FFT) used to predict the propagation of ultrasound waves in the far-field area [7]. The directivity function is achieved by applying the 2D FFT on the aperture function of the array [14].

\section{RESUlts AND DISCUSSION}

\section{A. Simulation Results}

The three sparse array configurations shown in Figure 1 were first modelled in MATLAB using a 2D FFT to predict the directivity functions as shown in Figure 2(b). As explained in Section II.A, for each configuration, a different number of parameters were required to design the array structure. A set of candidate values were created for each parameter. For the purposes of comparison, the parameters common to all array configurations were set to have the same candidate values for all the array configurations. Table I lists the parameters and corresponding candidate value intervals.

TABLE I. PARAMETERS AND CANDIDATE VALUES FOR EACH ARRAY CONFIGURATION.

\begin{tabular}{|c|c|c|}
\hline Parameters & Interval & Array Type \\
\hline Element radius & $\begin{array}{c}0.7: 0.05: 1.5 \\
(\mathrm{~mm})\end{array}$ & $\begin{array}{c}\text { All three array } \\
\text { configurations }\end{array}$ \\
\hline $\begin{array}{c}\text { Minimum gap between two } \\
\text { elements within the array }\end{array}$ & $\begin{array}{c}0.4: 0.05: 1 \\
(\mathrm{~mm})\end{array}$ & $\begin{array}{c}\text { All three array } \\
\text { configurations }\end{array}$ \\
\hline $\begin{array}{c}\text { Number of elements within each } \\
\text { log spiral arm }\end{array}$ & $3: 1: 10$ & Log Spiral Array \\
\hline Number of log spiral arms & $7: 2: 19$ & Log Spiral Array \\
\hline Constant parameter $b$ & $1.2: 0.1: 1.5$ & Log Spiral Array \\
\hline
\end{tabular}

By iterating through all candidate parameter values for each configuration, all possible sparse array patterns have been simulated using (1), (2), (3) and (4). For each array configuration, the PSL and ISLR were calculated and stored. Further analysis selected the design which minimized both parameters. Unfortunately, the array layout with the lowest PSL value had a relatively high ISLR value when compared to other patterns which have similar PSL values. Thus, instead of selecting the layout with the lowest PSL, a configuration with similar PSL but lower ISLR was selected for each configuration. Other factors, such as the manufacturing complexity and practical realization feasibility are also taken into account. The PSL and ISLR values of the selected pattern for each array configuration are as shown in Table II. As shown in the Table, the log spiral array has the lowest PSL, while the sunflower spiral array performs the best in terms of ISLR. The log spiral array pattern is selected as the final design, with Figure 2 showing the configuration and its simulated directivity function. The parameters are: $a: 4.5 \mathrm{~mm} ; b: 1.2$; and $\theta: 27.69 \mathrm{deg}$. There are 6 elements within each arm.

TABLE II. PSL AND ISLR FOR EACH OPTIMIZED PATTERN

\begin{tabular}{|c|c|c|}
\hline Array Type & PSL (dB) & ISLR (dB) \\
\hline Random Array & -16.93 & 2.67 \\
\hline Sunflower Spiral Array & -17.38 & 0.58 \\
\hline Log Spiral Array & -19.33 & 2.71 \\
\hline
\end{tabular}

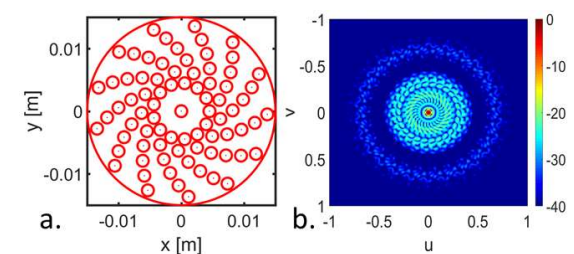

Fig. 2. (a.)The layout of the selected log spiral array. (b.)Directivity function of the selected log spiral array. The PSL is $-19.33 \mathrm{~dB}$ and the ISLR is $2.71 \mathrm{~dB}$.

\section{B. Manufacturing of Active Layers}

The selected log spiral array has been manufactured and an image of the device is shown in Figure 3. A jig has been made to hold the fibers in desired positions to facilitate fabrication. To make sure all the fibers are surrounded by polymer to ensure the piezocomposite can work properly, the volume fraction of each element is set to be $50 \%$. The piezoelectric materials used are $250 \mu \mathrm{m}$ diameter PZT5A fibers (Smart Material Corp., Sarasota, FL) and there are 28 fibers within each $0.95 \mathrm{~mm}$ array element. The jig with the fibers was placed inside a rectangular mold and CIBA-GEIGY CY221-HY956 epoxy poured into the mold and left to cure. The composite was then machined to $0.75 \mathrm{~mm}$ thickness to achieve the $2 \mathrm{MHz}$ operating frequency. $A g$ is used as the material for electrodes, with a thin layer of $\mathrm{Cr}$ evaporated first to improve adhesion to the active material.

\section{Characterization of Active Layers}

Before applying the log spiral electrode pattern, the fiber CECAT active layer was fully electroded and the impedance response of the active layer measured. The results are as shown in Figure 4. The measured resonance frequency for the entire active layer is $1986 \mathrm{kHz}$, which is very close to the desired 2 $\mathrm{MHz}$ working frequency. The coupling coefficient $\left(k_{t}\right)$ is 0.59 , which indicates the active layer should have appropriate energy conversion capability.

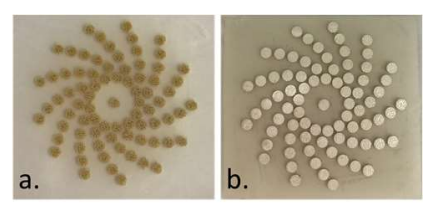

Fig. 3. The selected log spiral array manufactured using CECAT configuraion. (a.) Active layer before adding electrodes. (b.) Active layer after adding electrodes.

Next, the CECAT was electroded as a 2D array, as illustrated in Fig. 3(b), using evaporation through a mask and then the 
vibration of the active piezocomposite fiber elements was tested in air using a 3D Laser Doppler Vibrometer (LDV). The active layer was placed on a platform which is perpendicular to the laser source can be moved in the lateral directions. For each array element tested, the active layer was moved by the platform and scanned using the LDV with only one element being fired at each time to evaluate the cross-talk between the elements. The whole array surface was sampled into a set of points corresponding to the moving step of the platform. The data was then processed using Huygens' theory to generate the predicted beam profile of the active layer. The results are as shown in Figure 5. The beam profile generated from the experimental data has similar shape to the simulated result. The PSL of the experimental results is $-12.8 \mathrm{~dB}$. The ISLR of the experimental results is $10.9 \mathrm{~dB}$ which is higher than the simulated ISLR $(2.71$ $\mathrm{dB}$ ). This is because different models are used to represent the elements. In the original simulation process, an element is modelled as a surface. Here the element is represented by a collection of measured points and the resolution of the LDV gives around 10 points within each element area. Figure 6(a) and (b) respectively show the directivity functions of the array simulated using a model to simulate having 10 and 20 points within each element. As shown in the figure, the side-lobe levels are increased when the number of points is decreased. The simulated ISLR is $6.26 \mathrm{~dB}$ for the 10 points model, and $9.53 \mathrm{~dB}$ for the 20 points model. Thus, if the spatial resolution is increased, the side-lobe levels will be reduced and become more similar to the simulation results as presented in Section III.A.

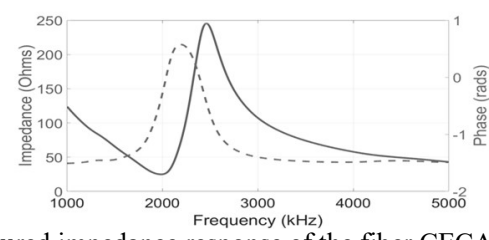

Fig. 4. The measured impedance response of the fiber CECAT active layer The solid line represents the impedance, while the dash line represents the phase. The electriccal resonance frequency is $1986 \mathrm{kHz}$.

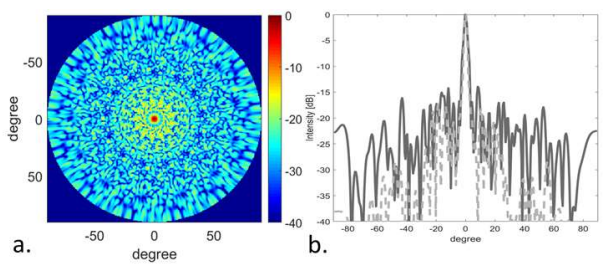

Fig. 5. (a.) The directivity function generated using measured data. (b.) Directivity functions which are generated using the experimental results (the solid line) and the simulated results (the dash line) in the central $\mathrm{x}-\mathrm{z}$ plane.

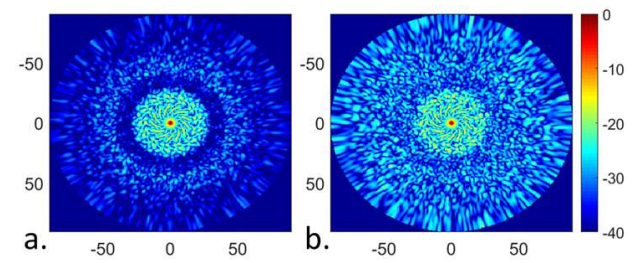

Fig. 6. Simulated directivity functions of the log spiral array using the second model. Each element is represented by (a.) 20 points and (b.) 10points.

\section{CONCLUSION}

Three aperiodic sparse array configurations have been studied in this project. The simulation results show that the log spiral array has an advantage in terms of minimizing PSL while the sunflower spiral array has lower ISLR. A log spiral array pattern has been selected as a compromise between optimal PSL and ISLR values. The active layer with selected array configuration has been manufactured using a fiber CECAT structure. The impedance response of the active layer shows it has appropriate $k_{t}$ and works at the desired design frequency of $2 \mathrm{MHz}$. One thing noticed from the simulation is that, PSL of the log spiral array configuration can be further reduced with tighter element patterns. However, the tighter element patterns would present a significant challenge for the current manufacturing process, especially for the fiber CECAT configuration. This is due to the fact that fibers which belong to neighboring elements will likely be overlap or touch as the fibers cannot stay straight in the jig. This challenge could be solved if the fiber-epoxy composite can be machined as a tube before inserting into the jig. In summary, the prototype active layer has desired performance in terms of the impedance response and the PSL. The measured ISLR seems to be much higher than predicted, but this is caused by the use of different models for data processing and the low spatial resolution used in the LDV test.

\section{REFERENCES}

[1] R. Aaslid, T. M. Markwalder, and H. Nornes, "Non-invasive Transcranial Doppler Ultrasound Recording of Flow Velocity in Basal Cerebralarteries," Journal of Neurosurgery, vol. 57, pp. 769-774, 1982.

[2] G. Tsivgoulis and A. V. Alexandrov, "Ultrasound-enhanced thrombolysis in acute ischemic stroke: Potential, failures, and safety," Neurotherapeutics, vol. 4, pp. 420-427, Jul 2007.

[3] J. Dziewierz, "2D ultrasonic phased arrays for quantitative characterisation of complex defects", PhD, University Strathclyde, 2015.

[4] B. D. Lindsey, E. D. Light, H. A. Nicoletto, E. R. Bennett, D. T. Laskowitz, and S. W. Smith, "The Ultrasound Brain Helmet: New Transducers and Volume Registration for In Vivo Simultaneous MultiTransducer 3-D Transcranial Imaging," IEEE Trans UFFC, vol. 58, pp. 1189-1202, 2011.

[5] H. Azhari, Basics of biomedical ultrasound for engineers, 1st ed. Hoboken, N.J.: Wiley, 2010, p. 313.

[6] G. Harvey, A. Gachagan, J. W. Mackersie, T. Mccunnie and R. Banks, "Flexible ultrasonic transducers incorporating piezoelectric fibres," in IEEE Transactions on Ultrasonics, Ferroelectrics, and Frequency Control, vol. 56, no. 9, pp. 1999-2009, September 2009.

[7] A. Tweedie, "Spiral 2D Array Designs for Volumetric Imaging", PhD, University of Strathclyde, 2011.

[8] O. Martínez-Graullera, C. Martín, G. Godoy and L. Ullate, "2D array design based on Fermat spiral for ultrasound imaging", Ultrasonics, vol. 50, no. 2, pp. 280-289, 2010.

[9] A. Ramalli, E. Boni, A. S. Savoia and P. Tortoli, "Density-tapered spiral arrays for ultrasound 3-D imaging," in IEEE Trans on UFFC, vol. 62, no. 8, pp. 1580-1588, 2015.

[10] M. Viganó, G. Toso, G. Caille, C. Mangenot and I. Lager, "Sunflower Array Antenna with Adjustable Density Taper", International Journal of Antennas and Propagation, vol. 2009, pp. 1-10, 2009.

[11] L. Lic. Mirko Panozzo Zénere, "SAR IMAGE QUALITY ASSESMENT", Master Degree, Universidad Nacional de Córdoba, 2012.

[12] B. B. Baker and E. T. Copson, The Mathematical Theory of Huygens' Principle. American Mathematical Soc., 2003. 28

[13] E. Franco, M. Andrade, J. Adamowski and F. Buiochi, "Acoustic beam modeling of ultrasonic transducers and arrays using the impulse response and the discrete representation methods", Journal of the Brazilian Society of Mechanical Sciences and Engineering, vol. 33(4), pp. 408-416, 2011.

[14] L. Ziomek, Funfamentals of acoustic field theory and space-time signal processing. Boca Raton: CRC Press, pp. 416, 1995 